\title{
C-Terminal Maturation Fragments of Presenilin 1 and 2 Control Secretion of APP $\alpha$ and AB by Human Cells and Are Degraded by Proteasome
}

\author{
Cristine Alves da Costa, Karine Ancolio, and Frédéric Checler \\ Institut de Pharmacologie Moléculaire et Cellulaire du CNRS, \\ Valbonne, France \\ Communicated by P. Chambon. Accepted February 15, 1999.
}

\begin{abstract}
Background: Most early-onset forms of Alzheimer's disease are due to missense mutations located on two homologous proteins named presenilin 1 and 2 (PS1 and PS2). Several lines of evidence indicate that PS1 and PS2 undergo various post-transcriptional events including endoproteolytic cleavages, giving rise to $28-30 \mathrm{kD} \mathrm{N}$ terminal (NTF) and 18-20 kD C-terminal (CTF) fragments that accumulate in vivo. Whether the biological activity of presenilins is borne by the processed fragments or their holoprotein precursor remains in question. We have examined the putative control of $\beta$ APP maturation by CTF-PS1/PS2 and the catabolic process of the latter proteins by the multicatalytic complex, proteasome.

Materials and Methods: We transiently and stably transfected HEK293 cells with CTF-PS1 or CTF-PS2 cDNA. We examined these transfectants for their production of $A \beta 40, A \beta 42$, and APP $\alpha$ by immunoprecipitation using specific polyclonals. The effect of a series of proteases inhibitors on the immunoreactivity of CTF-
\end{abstract}

PS1/PS2 was examined by Western blot. Finally, the influence of proteasome inhibitors on the generation of $\beta A P P$ fragments by CTF-expressing cells was assessed by combined immunoprecipitation and densitometric analyses.

Results: We showed that transient and stable transfection of CTF-PSI and CTF-PS2 cDNAs in human cells leads to increased secretion of APP $\alpha$ and $A \beta$, the maturation products of $\beta$ APP. Furthermore, we demonstrated that two proteasome inhibitors, lactacystin and Z-IE(Ot$\mathrm{Bu}$ )A-Leucinal, prevent the degradation of both CTFs. Accordingly, we established that proteasome inhibitors drastically potentiate the phenotypic increased production of APP $\alpha$ and A $\beta$ elicited by CTF-PS1/PS2.

Conclusion: Our data establish that the C-terminal products of PS1 and PS2 maturation exhibit biological activity and in particular control $\beta$ APP maturation upstream to $\alpha$-and $\beta / \gamma$-secretase cleavages. This function is directly controlled by the proteasome that modulates the intracellular concentration of CTFs.

\section{Introduction}

Familial forms of Alzheimer's disease are due to inherited mutations on genes, the loci of which have been identified on chromosomes 21, 14, and 1 (for review see ref. 1 ). The gene products of chromosomes 1 and 14 have been shown to be responsible for most early-onset forms of Alzhei-

Address correspondence and reprint requests to: Dr. F. Checler, Institut de Pharmacologie Moléculaire et Cellulaire du CNRS, UPR 41 1, 660 Route des Lucioles, Sophia Antipolis, 06560 Valbonne, France. Phone: (33) 4939577 60; Fax: (33) 4939577 04/08; E-mail: checler@ipmc.cnrs.fr mer's disease and have been identified as presenilins 2 and 1 (PS2 and PS1), respectively (2-4). PS1 and PS2 are highly homologous transmembrane proteins $(5,6)$ located mainly in the endoplasmic reticulum (7-11) and Golgi apparatus $(10,11)$. These proteins undergo various posttranscriptional modifications (for reviews see refs. 12,13) and are particularly susceptible to endoproteolytic cleavages giving rise to $\mathrm{N}$-terminal (NTF) and C-terminal (CTF) fragments (1417). This cleavage by an as-yet unknown protease seems to be a major event since intact PS 
holoproteins are poorly detectable in the brain of transgenic mice and affected patients and the two proteolytic fragments appear to accumulate with a $1 / 1$ stochiometry (15). Interestingly, NTF anf CTF undergo phosphorylation events $(9,18)$, behave as targets of caspase-mediated proteolysis (19-21), and can physically interact $(12,13)$. Whether these post-transcriptional events reflect regulatory mechanisms aimed at modulating putative NTF and CTF biological activity remains to be established.

We have examined whether the expression of CTF-PS1/PS2 modulates the processing of $\beta$ APP in HEK293 cells. We show that both CTFPS1 and CTF-PS2 expression leads to increased secretion of $A \beta$ and APP $\alpha$. Furthermore, we establish that both PS maturated fragments are catabolized by the proteasome. Accordingly, two proteasome inhibitors drastically potentiate the CTF-mediated increase in the recovery of both $\beta A P P$ maturation products. Altogether, our data indicate that CTF-PS1 and CTF-PS2 are biologically active and in particular, control $\beta$ APP maturation. This function appears to be directly modulated by the proteolytic catabolism elicited by the proteasome.

\section{Materials and Methods}

\section{Design of CTF-PS1 and CTF-PS2 CDNAS}

The cDNA encoding CTF-PS1 (ct-1) was engineered by introducing the Kosak sequence of PS1 upstream of the ATG codon encoding the PS1 methionine in position 292 (oligo: 5'-CATAGG-ATC-CGT-TGC-TCC-AAT-GGT-GTG-GTTGGT-GAA-TAT-GGC-AGA-A-3'). An additional $B a m H 1$ restriction site was also added, adjacent to the Kosak sequence for further subcloning of the construction in pcDNA3 (ct-1). The cDNA construction coding for CTF-PS2 (ct-2) was engineered similarly, with the Kozak sequence of PS2 upstream of the ATG codon encoding the methionine in position 298 and an additional Kpn restriction site adjacent to the Kozak sequence (oligo: 5'-ATC-TGG-TAC-CGG-CAGGGC-TAT-GGT-GTG-GAC-GGT-TGG-CAT-GGCGAA-G-3').

\section{Stable Transfections of HEK293 Cells}

HEK293 cells were grown as previously described (22). Stable transfectants were obtained by calcium phosphate precipitation with $1 \mu \mathrm{g}$ of empty pcDNA $_{3}$ vector, ct-1, or ct-2 and called
Mock, CPS1, and CPS2, respectively. CPS1 and CPS2 were identified after Western blot analysis of electrophoresed proteins by means of the $\alpha$ PS1Loop or $\alpha$ PS2Loop antibodies (15) as described previously $(23,24)$. HEK293 cells expressing wild-type- $\beta A \mathrm{AP}_{751}$ (referred to as WT) were obtained as described previously (22).

\section{Transient Transfections of HEK293 Cells}

Mock or WT stable transfectants were transiently transfected with $2 \mu \mathrm{g}$ of ct-1, ct-2, or empty vector by means of DAC30 according to manufacturer recommendations (Eurogentec). Transfection efficiency was checked by Western blot with $\alpha$ PS 1Loop or $\alpha$ PS2Loop antibodies. Analysis of $\beta$ APP maturation products was performed 48 to $72 \mathrm{hr}$ after transfection.

\section{Immunoprecipitation and Detection of $A P P \alpha$ and $A \beta$}

Transiently or stably transfected cells were maintained in the above F12/DMEM-supplemented medium then washed, and secretion of APP $\alpha$ and $A \beta$ was initiated for $5 \mathrm{hr}$ at $37^{\circ} \mathrm{C}$ in the absence or with presence of proteasome inhibitors. Aliquots of media were recovered, diluted in an equal volume of RIPA buffer, and incubated overnight with a 3000-fold dilution of 207 antibody (APP $\alpha$ ) or with a 350-fold dilution of FCA18 (total $\mathrm{A} \beta$ ) as previously described (25). Samples were centrifuged, and the pellets were washed three times with RIPA $1 \times$ containing $\mathrm{NaCl}(350 \mathrm{mM})$, rinsed with TBS buffer, then resuspended in the loading buffer, electrophoresed on $8 \%$ SDS-PAGE (APP $\alpha$ ) or Tris-tricine gels $(A \beta)$, and Western blotted for 1-3 hr. Nitrocellulose sheets were capped with skim milk ( $5 \%$ in TBS buffer) and exposed overnight to a 200fold dilution of mAb10D5C (APP $\alpha$ ) or $1 \mu \mathrm{g} / \mathrm{ml}$ of WO2 (A $\beta)$ antibodies. The nitrocellulose sheets were rinsed with TBS buffer then incubated with adequate anti-IgGs, revealed, and quantified as previously described (25).

\section{Western Blot Analysis of $\beta A P P$ and CTFs Immunoreactivity}

Stably transfected cells were treated as above then lysed in $50 \mathrm{mM}$ Tris- $\mathrm{HCl}, \mathrm{pH} 7.5$, containing $150 \mathrm{mM}$ of $\mathrm{NaCl}$ and protease inhibitors $(5 \mathrm{mM}$ EDTA, $1 \mathrm{mM}$ leupeptin, $1 \mu \mathrm{M}$ pepstatin and 1 $\mathrm{mM}$ AEBSF). Analysis for $\beta$ APP and CTF-PS $1 /$ CTF-PS2 contents was performed by means of BR188 antibody or $\alpha$ LoopPS1/PS2 as described previously (26). 

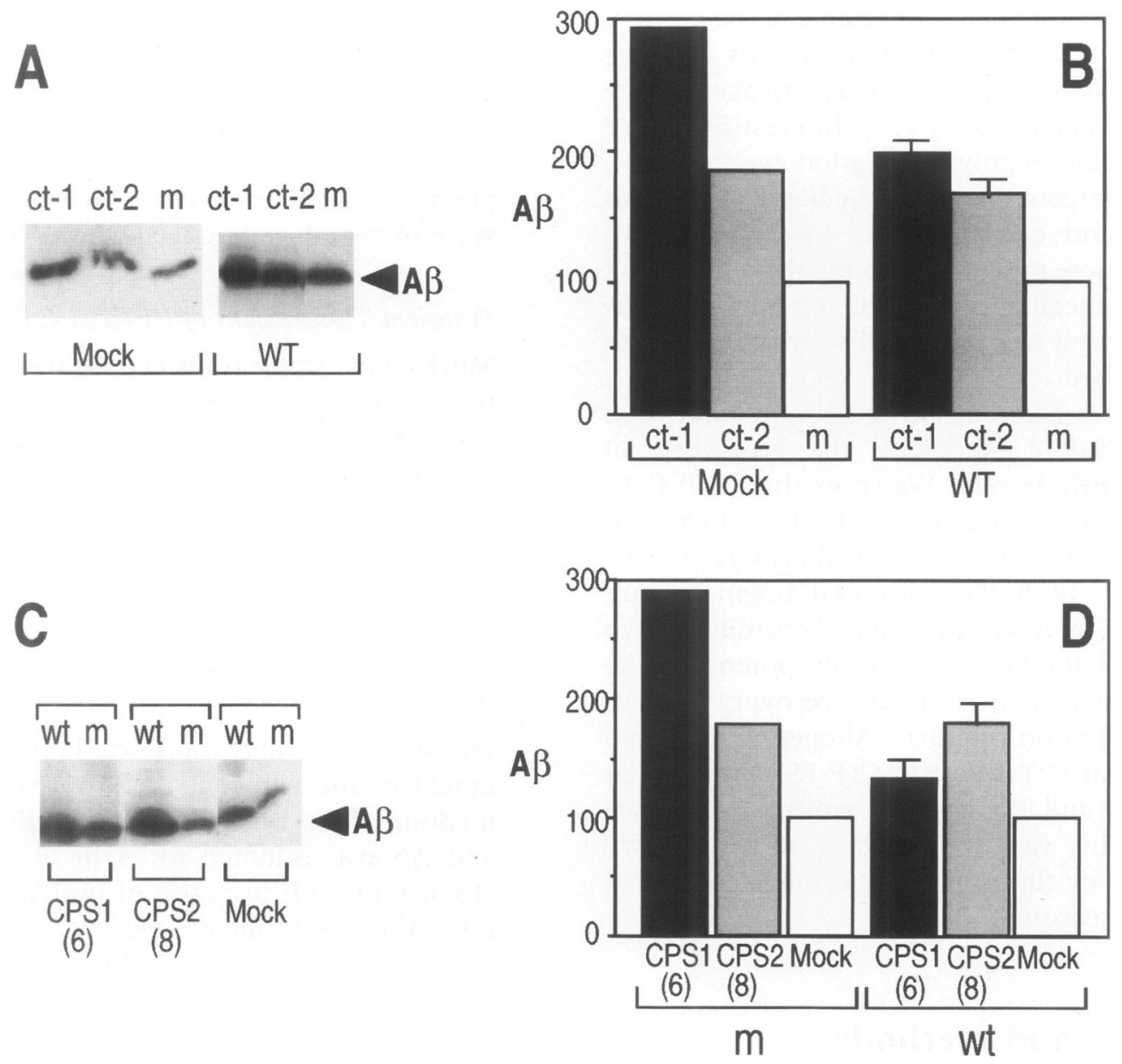

Fig. 1. Influence of CTF-PS1 and CTF-PS2 on $\mathbf{A} \boldsymbol{\beta}$ secretion. Stable transfectants expressing wt-

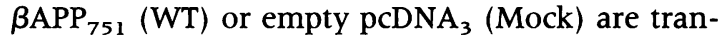
siently transfected with empty pcDNA3 $(\mathrm{m})$ or coding for CTF-PS1 (ct-1) or CTF-PS2 (ct-2) fragments (A). Secreted $A \beta$ was immunoprecipitated with FCA18 antibody then detected with mAbWO2 as described in Materials and Methods. Bars in B correspond to densitometric analysis of secreted $A \beta$ (normalized for ct-1 and ct-2 expression) and are expressed as the percent of control corresponding to Mock and WT cells transiently transfected with empty pcDNA $(\mathrm{m})$. Values are the mean \pm SEM of 3

\section{Antibodies}

FCA18 (27) and WO2 (28) specifically interact with the $\mathrm{N}$-terminus of $\mathrm{A} \beta$. The 207 antibody (donated by Drs. M. Savage and B. Greenberg, Cephalon, Westchester, NY) recognizes the Nterminus of $\beta$ APP and APP $\alpha$. 10D5C (provided by Dr. D. Schenk, Athena Neurosciences) specifically recognizes the C-terminus of APP $\alpha$. $\alpha$ PS 1Loop and $\alpha$ PS2Loop (provided by Dr. G. Thinakaran, Johns Hopkins University, Baltimore) specifically interact with the hydrophilic

loop of PS1 and PS2 located between their predicted sixth and seventh transmembrane domains. BR188 (supplied by Dr. M. Goedert, Cambridge, England) recognizes the C-terminus of mature and immature $\beta$ APP.

\section{Results}

Stably mock-transfected HEK293 cells (Mock) were transiently transfected with empty pcDNA3 (m), ct-1, or ct-2 (Fig. 1A, left panel). CTF-PS 1 

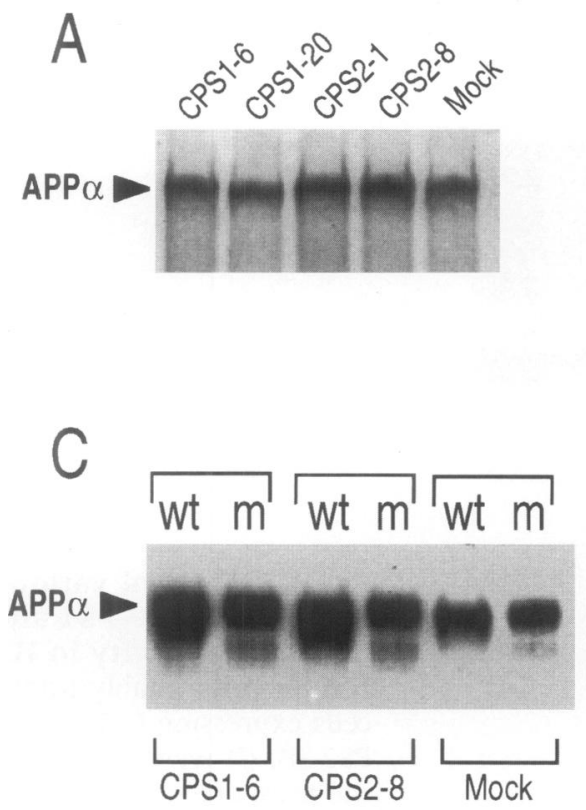

Fig. 2. Influence of CTF-PS1 and CTF-PS2 overexpression on APP $\alpha$ secretion. Stably transfected HEK293 cells overexpressing CTF-PS1 (CPS1-6 and CPS1-20), CTF-PS2 (CPS2-1 and CPS28), or empty pcDNA ${ }_{3}$ (Mock) were examined for APP $\alpha$ secretion from endogenous $\beta$ APP (A, B) or after transient transfection (C, D) with $\beta$ APP75 1 (wt) or pcDNA3 (m). APP $\alpha$ was detected after immunoprecipitation with 207 antibody and western blot with MAB10D5C as described in Materials and

and CTF-PS2 increased the recovery of total secreted $A \beta$ (Fig. 1A, left panel) by about 200 to $300 \%$ (Fig. IB) over control (Mock/m) cells, indicating that transient transfection of ct-l and ct- 2 modulates the $A \beta$ production derived from endogenous $\beta$ APP. The same phenotypic increase in $A \beta$ secretion was observed (Fig. 1A, right panel) with stably transfected wt- $\beta A \mathrm{AP}_{751^{-}}$ expressing cells (WT) transiently transfected with ct- 1 and ct-2 (about $200 \%$ over control $\mathrm{WT} / \mathrm{m})$.

To further document the influence of the overexpression of CTF-PS $1 / 2$ on the $\beta / \gamma$-derived product of $\beta$ APP maturation, clones stably mocktransfected (Mock) or overexpressing CTF-PS 1 (CPS1) and CTF-PS2 (CPS2) were transiently transfected with empty pcDNA ${ }_{3}$ (m) (Fig. 1C). Both CTF-expressing clones secreted higher amounts of $\mathrm{A} \beta$ than $\mathrm{Mock} / \mathrm{m}$ control cells (Fig. ID). Transient transfection of Mock, CPS1, and CPS 2 clones with wt- $\beta A P P_{751}$ cDNA (wt) led to increased $A \beta$ secretion, the production of which was higher in CPS1 and CPS2 than in Mock-transfected cells (Fig. 1C, D).
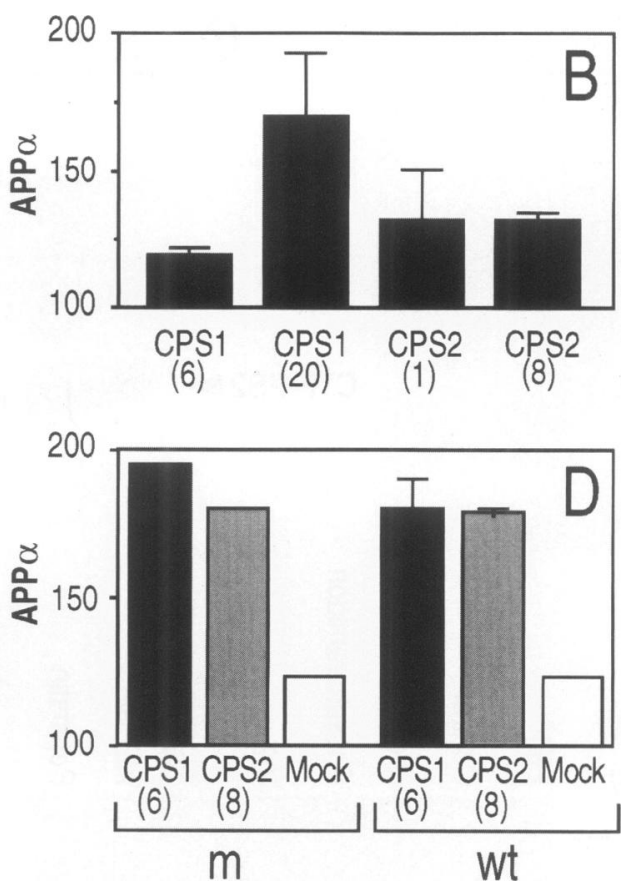

Methods. Bars in B and D represent the APP $\alpha$ densitometric analysis of experiments A (normalized for CTF-PS1/PS2 expressions) and C (normalized for wt expression), respectively. Values are expressed as the percent of control APP $\alpha$ secretion obtained with Mock cells (B) or Mock-transfected cells (D) transiently transfected with empty vector $(\mathrm{m})$ or wt$\beta_{A P P}{ }_{751}(w t)$. Values are the means \pm SEM of independent experiments.

We examined the putative influence of CTFPS1 and CTF-PS2 expression on the secretion of the $\alpha$-secretase-derived physiological product of $\beta A P P$ maturation, APP $\alpha$. Two independent clones of CPS1 and CPS2 produced higher amounts of APP $\alpha$ than Mock-transfected cells (Fig. 2A, B). Transient transfection of Mock, CPS1, and CPS2 (Fig. 2C) with empty pcDNA3 (m) or wt- $\beta A P P_{751}$ cDNA (wt) confirmed that the CTF-PSs-expressing cells secrete more APP $\alpha$ than the Mock-transfected cells (Fig. 2D).

The effect of the proteasome inhibitor Z-IE(Ot-Bu)A-Leucinal on CTF-PSI and CTFPS2 immunoreactivity in stable transfectants was also examined. Figure 3 indicates that immunoreactivity of both CTF-PS1 (Fig. 3A) and CTF-PS2 (Fig. 3B)is highly enhanced by Z-IE(Ot-Bu)ALeucinal. The involvement of the proteasome in CTF degradation is further demonstrated by the enhancement of CTF immunoreactivity upon treatment with lactacystin, a very potent and selective proteasome blocker (Fig. 3C). It should be noted that a smir of high-molecular-weight proteins is detectable upon proteasome inhibi- 


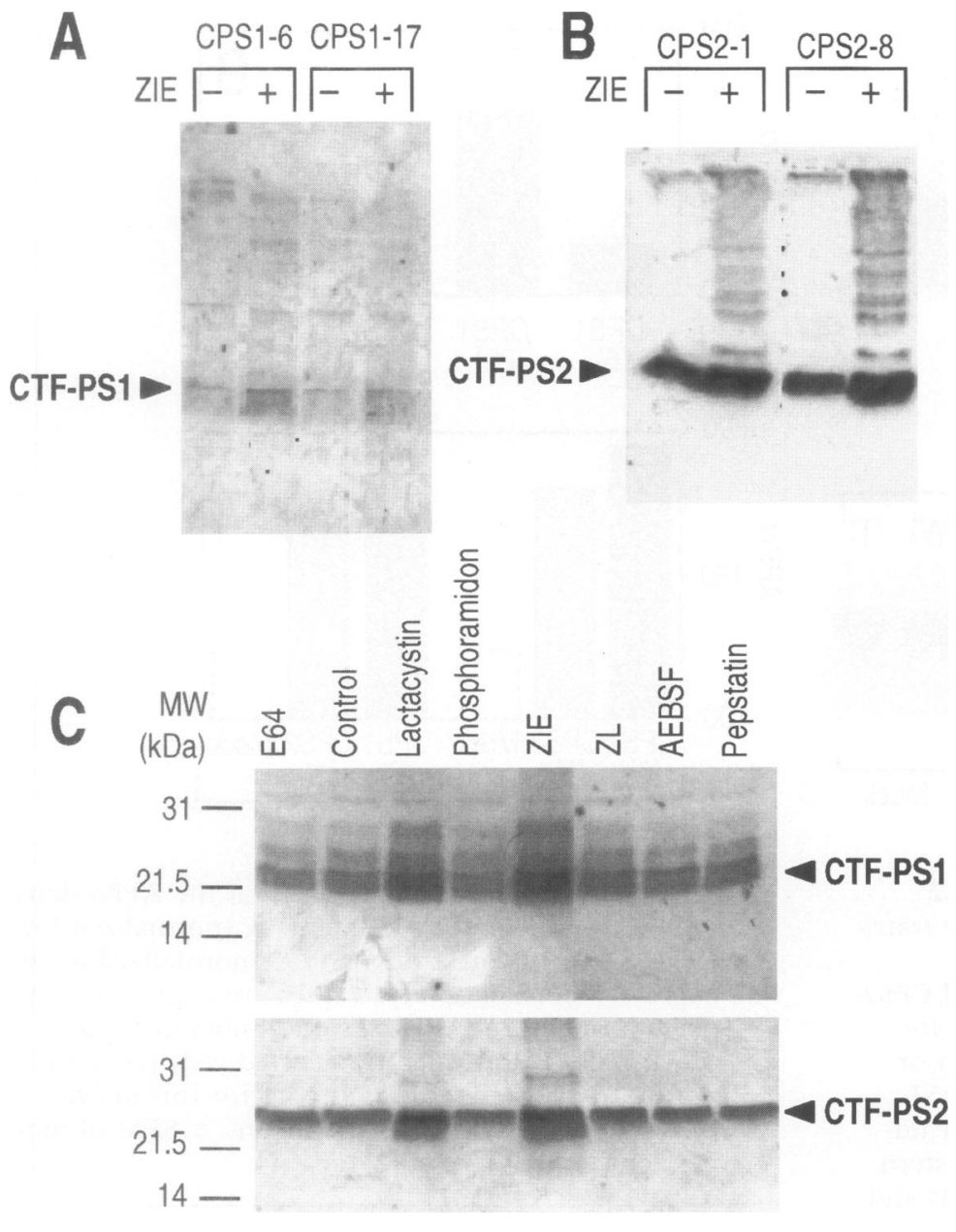

Fig. 3. Effect of various protease inhibitors on CTF-PS1 and CTF-PS2 immunoreactivity in HEK293 transfected cells. Stably transfected HEK293 cells expressing CTF-PS 1 (A, C) or CTFPS2 $(B, C)$ were incubated for $5 \mathrm{hr}$ in the presence of the proteasome inhibitors Z-IE(Ot-Bu)A-leucinal (ZIE, $2.5 \times$ $\left.10^{-5} \mathrm{M}\right)$ and lactacystin $\left(5 \times 10^{-6} \mathrm{M}\right)$ or with trans-epoxysuccinyl-L-leucylamido(4-guanidino) butane $\left(\mathrm{E} 64,10^{-4} \mathrm{M}\right)$, 4-(2-amido-ethyl) benzenesulfonyl fluoride (AEBSF, $10^{-4} \mathrm{M}$ ), phosphoramidon $\left(10^{-5} M\right)$, pepstatin $A\left(10^{-5} M\right)$, and Z-L-Leucinal (ZL, $\left.2.5 \times 10^{-5} \mathrm{M}\right)$. Cells were lysed, then immunoreactivity of CTF-PS was revealed with $\alpha$ PS ILoop and $\alpha$ PS2Loop antibodies as described in Materials and Methods. tion (see Fig. 3B), which could reflect protection of ubiquitinated forms of CTF as was shown for presenilin 1 (23) and presenilin 2 (29).

Other protease blockers unable to inhibit the proteasome activity do not modify CTF-PS1/2 recovery (Fig. 3C). Thus, Z-L-Leucinal (calpain and cathepsin B inhibitor), E64 (thiol and serine protease inhibitor), AEBSF (serine protease inhibitor), phosphoramidon (endopeptidase 3.4.24.11 inhibitor), and pepstatin (acid protease inhibitor) do not protect CTF-PS1 and CTF-PS2 from degradation in HEK293 cells (Fig. 3C).

If CTF-PS $1 / 2$ degradation by the proteasome is physiologically relevant, one would expect to potentiate their stimulatory effect on $A \beta$ and APP $\alpha$ secretion upon proteasome inhibition. ZIE(Ot-Bu)A-Leucinal augments the APP $\alpha$ secretion triggered by CPS1 and CPS2 transfectants (Fig. 4A, B). This inhibitor also potentiates the secretion of $A \beta$ by wt- $\beta A P P_{751}$-expressing transfectants (WT) transiently transfected with ct-1 and ct-2 constructions (Fig. 4C, D). Therefore, it can be concluded that inhibition of the proteasome potentiates the effect of CTF-PSI and CTFPS2 on both $\alpha$ - and $\beta / \gamma$-secretase-derived products of $\beta$ APP maturation in HEK293 cells.

\section{Discussion}

We have previously established that the proteasome contributes to the maturation of endogenous $\beta$ APP in human cells. Thus, proteasome inhibitors increase the recovery of both $A \beta$ and APP $\alpha$ in naive HEK293 cells (22). This led us to hypothetize that a cellular intermediate located upstream to the $\alpha$ - and $\beta / \gamma$-secretases cleavages and behaving as substrate of the proteasome plays an important role in $\beta$ APP maturation. The search for such an effector led us to suggest that PS1 and PS2 could fulfill such a role. First, we showed that overexpression of PS1 (26) and PS2 (24) elicit an increased secretion of both $A \beta$ and $\operatorname{APP} \alpha$. Second, we demonstrated that PSl and 

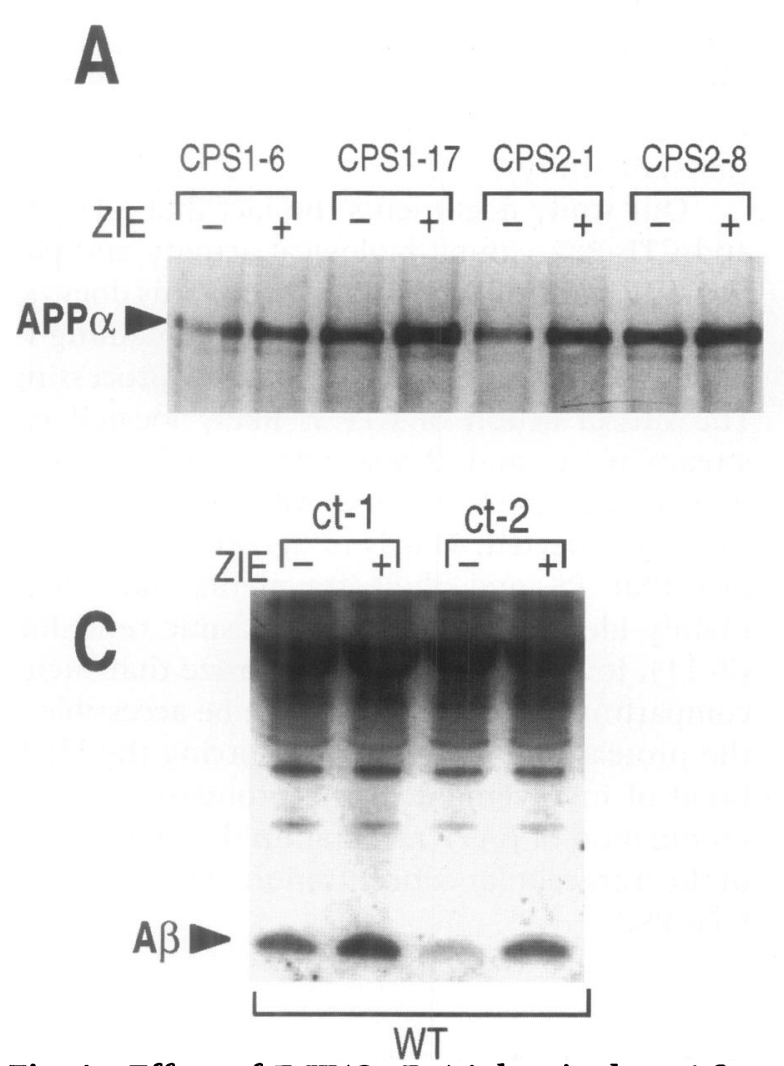

Fig. 4. Effect of Z-IE(Ot-Bu)A-leucinal on AB and APP $\alpha$ secretion by stably transfected HEK293 expressing CTF-PS1 and CTF-PS2. Stable transfectants overexpressing CTF-PS1 (CPS1-6 and CPS 1-17) and CTF-PS2 (CPS2-1 and CPS2-8) were grown as described in Materials and Methods then APP $\alpha$ secretion was initiated in the absence (-) or presence (+) of Z-IE(Ot-Bu)A-leucinal. APP $\alpha$ was immunoprecipitated after $5 \mathrm{hr}$ by means of 207 antibody and revealed with mAbloD5C (A). Stable transfectants overexpressing wt- $\beta$ APP75 1 (WT) were

PS2 behave as excellent substrates of the proteasome $(23,24)$, which is in agreement with other studies $(29,30)$. Third, we established that the proteasome inhibitors exacerbate the PS1/PS2induced increased recovery of both $\beta$ APP maturation products $(23,24)$. Interestingly, proteasome inhibitors also exacerbate the phenotypic alterations of $\beta$ APP maturation triggered by FAD-linked PS mutations $(23,24)$. Therefore, we suggested that PS 1 and PS 2 control $\beta$ APP maturation upstream to secretases, and that this function is directly modulated by catabolic events triggered by the proteasome.

Presenilins undergo several post-transcriptional modifications that include phosphorylation and proteolytic cleavages by unknown proteases as well as by caspase $3(12,13)$. Whether
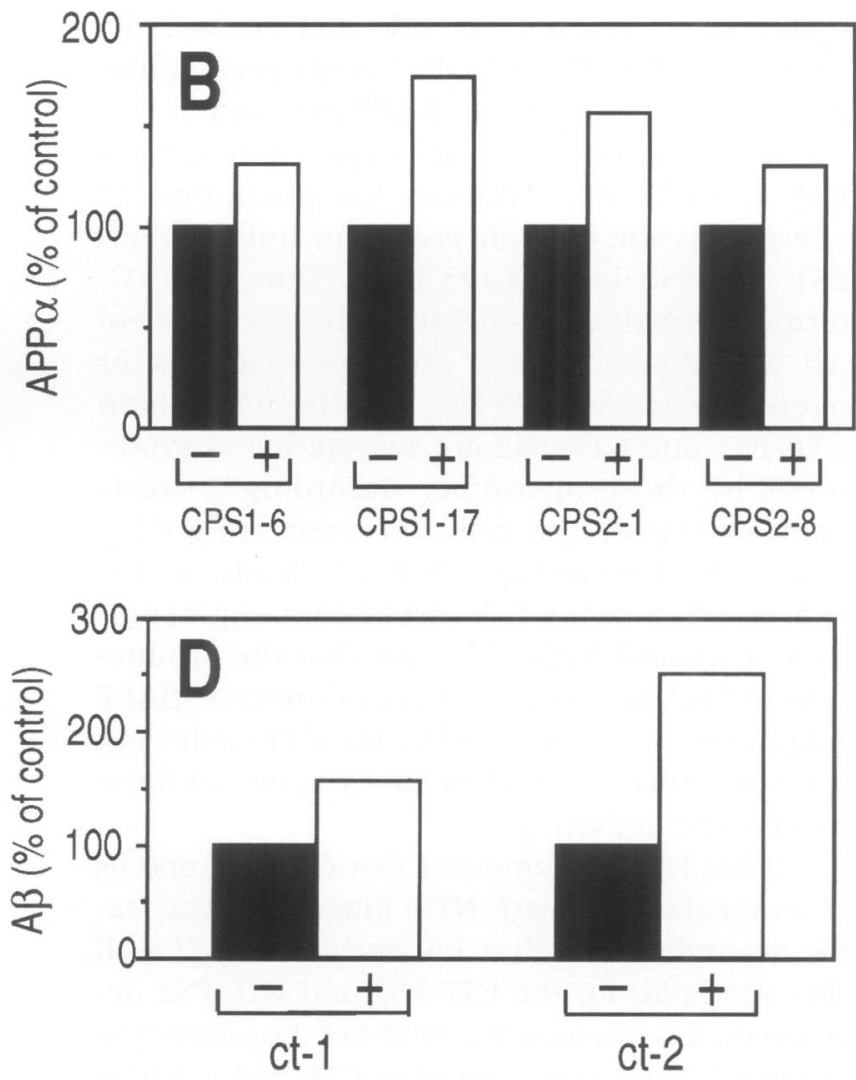

transiently transfected with CTF-PSI (ct-1) or CTFPS2 (ct-2) cDNAs. Secreted total A $\beta$ was obtained after immunoprecipitation with FCA 18 and revealed with mAbWO2 as described in Materials and Methods (C). Densitometric analyses of APP $\alpha$ (B) or A $\beta$ (D) [normalized for CTF-PS1/2 (A) or ct-1/2 (C)] secretions are expressed as the percent of control corresponding to secretion obtained in absence of inhibitor. Values are the mean of independent experiments carried out on the indicated independent clones.

the degradation products of PS1/PS2 correspond to intermediate or final catabolites en route to final clearance or, alternatively, to maturated fragments bearing biological activity is not yet established. However, the fact that these products accumulate in vivo in human and transgenic mice brains $(15,31)$ and are able to interact physically raised the possibility that $\mathrm{N}$ - and $\mathrm{C}$-terminal PS fragments could display a physiological function. In line with this hypothesis was the observation that the overexpression of a C-terminal fragment of PS2 that can be physiologically generated by proteolysis and alternative transcription was able to rescue HeLa cells from Fas- and TNF-induced apoptosis (32). Therefore, it was still questionable whether PS-related function was indeed due to PS holoproteins, their 
maturated fragments, or both, and particularly, whether CTF-PS 1 or CTF-PS2 could play a putative role in the control of $\beta$ APP maturation.

We show here that the expression of CTFPS1 and CTF-PS2 mimicks the phenotype of overexpression of their precursor holoproteins PS 1 and PS2 in HEK293 cells. Thus, both Cterminal fragments augment the secretion of $\mathrm{A} \beta$ and $\mathrm{APP} \alpha$ derived from endogenous or overexpressed wt- $\beta A \mathrm{APP}_{751}$. Furthermore, both CTF-PS 1 and CTF-PS2 are susceptible to proteolysis by the proteasome. Accordingly, treatment of transfected cells overexpressing CTFPSs with proteasome inhibitors leads to the exacerbation of the CTF-dependent augmentation of $\mathrm{A} \beta$ and $\mathrm{APP} \alpha$. The fact that the production of both $\alpha$ and $\beta / \gamma$-secretase-derived $\beta$ APP fragments are augmented by the CTFs rules out the possibility that CTF behaves as one of these proteolytic activities.

It has been documented that CTF-PSI and its $\mathrm{N}$-terminal counterpart (NTF) interact to form stable heterodimers with a 1:1 stochiometry (15). If this also holds for the CTF-PS2 and NTF-PS2 derivatives, it is questionable whether the phenotype observed when overexpressing CTF-PS2 mimicks the physiological function of endogenously produced CTF-PS2, since the 1:1 stochiometry of endogenous fragments theoretically preclude CTFs from interacting with endogenous NTFs-this fragment would not be available as a free entity. However, it should be noted that several studies have indicated that transient or stable transfection of PS2 led to apoptosis and that transfection of CTF-PS2 cDNA antagonized this effect in various cell systems, suggesting that overexpression of this fragment elicits a physiologically relevant phenotype (32).

Interestingly, Tomita and colleagues also recently examined the effect of overexpression of CTF-PS2 in stably transfected cells and concluded that this did not affect $\beta$ APP maturation (33). These discrepancies with our present work can likely be explained by several factors. The CTF constructions used in the Tomita study had different $\mathrm{N}$-termini from ours. Also, the effect of CTF expression was monitored on $\mathrm{x}-40 / 42$ species that likely include a major contribution of N-terminally truncated fragments derived from either pathogenic or also physiologic cleavages (33), whereas our antibodies were able to monitor total but genuine $A \beta$. Part of the data in Tomita's study concerns the effect of CTF on $x-40 / 42$ fragments derived from the C100 $\beta$ APP construction, the process- ing/routing of which could be different from full-length $\beta$ APP. Finally, it can be noted that the respective cells systems are totally distinct [COS and N2a (33) versus HEK293 cells (present study)].

Our study documents the fact that CTF-PS1 and CTF-PS2 exhibit biological activity and particularly, control $\beta$ APP maturation. This does not preclude the possibility that intact remaining PS holoproteins also play a role in $\beta$ APP processing. The site of action of CTF is likely located upstream to $\alpha$ - and $\beta / \gamma$-secretases cell compartments since both $\mathrm{A} \beta$ and $\mathrm{APP} \alpha$ secretions are similarly affected. This is in agreement with the fact that PS and their fragments have been mainly identified in the endoplasmic reticulum (7-11). It is interesting to emphasize that such a compartment has been shown to be accessible to the proteasome, therefore reinforcing the likelihood of involvement of the proteasome in the modulation of $\beta$ APP maturation through control of the intracellular concentration of CTF-PSI and CTF-PS2.

\section{Acknowledgments}

We thank Dr. D. Schenk (Athena Neuroscience, San Francisco, CA) for providing us with 10D5C antibodies. We sincerely thank Dr. G. Thinakaran (Johns Hopkins University, Baltimore, $M D$ ) for supplying us with the PS2 cDNA and $\alpha$ PS2Loop antibody. We are grateful to Dr. K. Beyreuther (Heidelberg, Germany) for providing us with WO2. We thank Drs. B. Greenberg and M. Savage for their kind supply of the 207 antibody. We thank J. Kervella for secretarial assistance. A.d.C. is recipient of a grant from the Fundação de Amparo a Pesquisa do Estado de São Paulo (FAPESP, Brazil). This work was supported by the Centre National de la Recherche Scientifique and the Institut National de la Santé et de la Recherche Médicale.

\section{References}

1. Tanzi RE, St. George-Hyslop P, and Gusella, JF. (1991) Molecular genetics of Alzheimer disease amyloid. J. Biol. Chem. 266: 20579-20582.

2. Sherrington R, Rogaev EI, Liang, Y, et al. (1995) Cloning of a gene bearing missense mutations in early-onset familial Alzheimer's disease. Nature 375: 754-760.

3. Levy-Lahad E, Wasco W, Poorkaj P, et al. (1995) 
Candidate gene for the chromosome 1 familial Alzheimer's disease locus. Science 269: 973-977.

4. Rogaev EI, Sherrington R, Rogaeva EA, et al. (1995) Familial Alzheimer's disease in kindreds with missense mutations in a gene on chromosome 1 related to the Alzheimer's disease type 3 gene. Nature 376: 775-778.

5. Doan A, Thinakaran, G, Borchelt DR, et al. (1996) Protein topology of presenilin 1. Neuron 17: 10231030.

6. Lehmann S, Chiesa R, Harris DA. (1997) Evidence for a six-transmembrane domain structure of presenilin 1. J. Biol. Chem. 272: 12047-12051.

7. Takashima A, Sato M, Mercken M, et al. (1996) Localization of Alzheimer-associated presenilin 1 in transfected COS-7 cells. Biochem. Biophys. Res. Commun. 227: 423-426.

8. Cook DG, Sung JC, Golde TE, et al. (1996) Expression and analysis of presenilin 1 in a human neuronal system: localization in cell bodies and dendrites. Proc. Natl. Acad. Sci. U.S.A. 93: 9223-9228.

9. Walter J, Capell A, Grünberg J, et al. (1996) The Alzheimer's disease-associated presenilins are differentially phosphorylated proteins located predominantly within the endoplasmic reticulum. Mol. Med. 2: 673-691.

10. Kovacs DM, Fausett HJ, Page KJ, et al. (1996) Alzheimer-associated presenilins 1 and 2: neuronal expression in brain and localization to intracellular membranes in mammalian cells. Nat. Med. 2: 224-229.

11. De Strooper B, Beullens M, Contreras B, et al. (1997) Phosphorylation, subcellular localization, and membrane orientation of the Alzheimer's disease-associated presenilins. J. Biol. Chem. 272: 3590-3598.

12. Mattson MP, Guo Q, Furukawa K, et al. (1998) Presenilins, the endoplasmic reticulum, and neuronal apoptosis in Alzheimer's disease. J. Neurochem. 70: 1-14.

13. Checler F. (1999) Presenilins. Mol. Neurobiol. (in press).

14. Ward RV, Davis JB, Gray CW, et al. (1996) Presenilin-l is processed into two major cleavage products in neuronal cell lines. Neurodegeneration 5: 293-298.

15. Thinakaran G, Borchelt DR, Lee MK, et al. (1996) Endoproteolysis of presenilin $\mathrm{l}$ and accumulation of processed derivatives in vivo. Neuron 17: 181190.

16. Baumann K, Paganetti PA, Sturchler-Pierrat C, et al. (1997) Distinct processing of endogenous and overexpressed recombinant presenilin 1. Neurobiol. Aging 18: 181-189.

17. Shirotani K, Takahashi K, Ozawa K, et al. (1997) Determination of a cleavage site of presenilin 2 protein in stably transfected SH-SY5Y human neuroblastoma cell lines. Biochem. Biophys. Res. Commun. 240: 728-731.
18. Seeger M, Nordstedt C, Petanceska S, et al. (1997) Evidence for phosphorylation and oligomeric assembly of presenilin 1. Proc. Natl. Acad. Sci. U.S.A. 94: $5090-5094$.

19. Loetscher $H$, Deuschle U, Brockhaus $M$, et al. (1997) Presenilins are processed by caspase-like proteases. J. Biol. Chem. 272: 20655-20659.

20. Kim TW, Pettingell WH, Jung YK, et al. (1997) Alternative cleavage of Alzheimer-associated presenilins during apoptosis by a caspase- 3 family protease. Science 277: 373-376.

21. Grünberg J, Walter J, Loetscher H, et al. (1998) Alzheimer's disease associated presenilin-l holoprotein and its 18-20 kDa C-terminal fragment are death substrates for proteases of the caspase family. Biochemistry 37: 2263-2270.

22. Marambaud P, Lopez-Perez E, Wilk S, et al. (1997) Constitutive and protein kinase C-regulated secretory cleavage of Alzheimer's $\beta$ amyloid precursor protein: different control of early and late events by the proteasome. J. Neurochem. 69: 2500-2505.

23. Marambaud P, Ancolio K, Lopez-Perez E, et al. (1998) Proteasome inhibitors prevent the degradation of familial Alzheimer's disease-linked presenilin 1 and trigger increased $A \beta 42$ secretion by human cells. Mol. Med. 4: 146-156.

24. Marambaud $P$, Alves da Costa $C$, Ancolio K, et al. (1998) Alzheimer's disease-linked mutation of presenilin 2 (N141I-PS2) drastically lowers APP $\alpha$ secretion: control by the proteasome. Biochem. Biophys. Res. Commun. 252: 134-138.

25. Marambaud $P$, Chevallier N, Ancolio $K$, et al. (1998) Post-transcriptional contribution of a cAMP-dependent pathway to the formation of $\alpha$ - and $\beta / \gamma$-secretases-derived products of $\beta$ APP maturation in human cells expressing wild type and Swedish mutated BAPP. Mol. Med. 4: 715723.

26. Ancolio K, Marambaud P, Dauch P, et al. (1997) $\alpha$-secretase-derived product of $\beta$-amyloid precursor protein is decreased by presenilin 1 mutations linked to familial Alzheimer's disease. J. Neurochem. 69: 2494-2499.

27. Barelli H, Lebeau A, Vizzavona J, et al. (1997) Characterization of new polyclonal antibodies specific for 40 and 42 aminoacid-long amyloid $\beta$ peptides: their use to examine the cell biology of presenilins and the immunohistochemistry of sporadic Alzheimer's disease and cerebral amyloid angiopathy cases. Mol. Med. 3: 695707.

28. Ida N, Johannes H, Pantel J, et al. (1996) Analysis of heterogeneous $\beta A 4$ peptides in human cerebrospinal fluid and blood by a newly developed sensitive Western blot assay. J. Biol. Chem. 271: 22908-22914.

29. Kim TW, Pettingell WH, Hallmark OG, et al. (1997) Endoproteolytic cleavage and proteasomal 
degradation of presenilin 2 in transfected cells. J. Biol. Chem. 272: 11006-11010.

30. Fraser PE, Levesque G, Yu G, et al. (1998) Presenilin 1 is actively degraded by the $26 \mathrm{~S}$ proteasome. Neurobiol. Aging 19: S19-S21.

31. Lee MK, Borchelt DR, Kim G, et al. (1997) Hyperaccumulation of FAD-linked presenilin 1 variants in vivo. Nat. Med. 3: 756-760.

32. Vito P, Ghayur T, and D'Adamio L, (1997) Generation of anti-apoptotic presenilin-2 polypeptides by alternative transcription, proteolysis, and caspase- 3 cleavage. J. Biol. Chem. 272: 28315-28320.

33. Tomita $\mathrm{T}$, Tokuhiro $\mathrm{S}$, Hashimoto $\mathrm{T}$, et al. (1998) Molecular dissection of domains in mutant presenilins 2 that mediate overproduction of amyloidogenic forms of amyloid $\beta$ peptides. Inability of truncated forms of PS2 with familial Alzheimer's disease to increase secretion of A $\beta 42$. J. Biol. Chem. 273: 2115321160 